\title{
ON THE RATIONALITY OF SOME MODULI SPACES RELATED TO POINTED TRIGONAL CURVES
}

\author{
G. CASNATI
}

\begin{abstract}
For each $g \geq 1$, let $\mathscr{T}_{g, n} \subseteq \mathcal{M}_{g, n}$ be the locus of points corresponding to curves carrying a base-point-free $g_{3}^{1}$. Here we give a proof that $\mathscr{T}_{g, n}$ is rational if $g \geq 4$ and $1 \leq n \leq 2 g+7$.
\end{abstract}

\section{Introduction and notation}

Let $\mathscr{M}_{g, n}$ be the (coarse) moduli space of projective, smooth and connected curves of genus $g$ with $n$ marked points defined over the field C, i.e., ordered $(n+1)$-tuples of the form $\left(C, p_{1}, \ldots, p_{n}\right)$ where $C$ is a smooth and connected curve of genus $g$ and $p_{1}, \ldots, p_{n} \in \mathrm{C}$ are pairwise distinct points.

Let $\mathscr{T}_{g, n} \subseteq \mathcal{M}_{g, n}$ be the locus of points $\left(C, p_{1}, \ldots, p_{n}\right)$ such that $C$ carries a base-point-free $g_{3}^{1}$. If $g \leq 4$, the locus $\mathscr{T}_{g, n}$ is dense inside $\mathscr{M}_{g, n}$ (at least when $\mathscr{M}_{g, n}$ actually exists) while, if $g \geq 5$, the closure of $\mathscr{T}_{g, n}$ is strictly contained in $\mathcal{M}_{g, n}$. We say that the points of $\mathscr{T}_{g, n}$ represent pointed trigonal curves, hence we look at $\mathscr{T}_{g, n}$ as a coarse moduli space for smooth and connected $n$-pointed trigonal curves of genus $g$.

From now on we will assume $g \geq 5$. It is well-known that the locus $\mathscr{T}_{g, n}$ is irreducible of dimension $2 g+1+n$. For the case $n=0$ see [1], Theorem 5.3, Formula (2.3) and the references therein. For the case $n>1$, see Section 3 for an elementary proof.

It thus makes sense to ask whether such a locus is rational, i.e., whether there is a birational isomorphism $\mathscr{T}_{g, n} \approx \mathrm{C}^{\oplus 2 g+1+n}$. The rationality of $\mathscr{T}_{g, 0}$ has been proved in Theorem 5 in [12], when $g \equiv 2(\bmod 4)$, and, when $g$ is odd, in the very recent paper [7].

In the present paper we focus our attention to the case of pointed trigonal curve, proving the following theorem.

THEOREM 1.1. The locus $\mathscr{T}_{g, n} \subseteq \mathcal{M}_{g, n}$ of trigonal n-pointed curves of genus $g$ is rational if $g \geq 5$ and $1 \leq n \leq 2 g+7$. 
This theorem can be viewed as a natural generalisation of the Main Theorem of [3], when $g=4$ and it is already known for $n=1$ and $g$ odd (see again [12]). Our approach is based on an easy improvement of the description of plane models of trigonal curves of odd genus given in [12].

It is interesting to notice that an analogous result is known for the moduli space $\mathscr{H}_{g, n}$ of $n$-pointed hyperelliptic curves, i.e., curves carrying a complete base-point-free $g_{2}^{1}$. More precisely, the rationality of $\mathscr{H}_{g, n}$ has been proved in [2] when $1 \leq n \leq 2 g+8$.

With the same methods, it is also possible to give a quick and easy proof of the rationality of $\mathscr{T}_{5,0}$ and $\mathscr{T}_{7,0}$ alternative to the one in [7] (and indeed such a proof was part of the first submitted version of this paper).

\subsection{Notation}

We work over the complex field $\mathrm{C}$. We denote by $\mathrm{GL}_{k}$ the general linear group of $k \times k$ matrices with entries in $\mathrm{C}$ and by $\mathrm{PGL}_{k}$ the projective linear group, i.e., $\mathrm{GL}_{k}$ modulo the subgroup of scalar matrices.

If $V$ is a vector space, then we denote by $\mathrm{P}(V)$ the corresponding projective space. In particular, we set $\mathrm{P}_{C}^{n}:=\mathrm{P}\left(\mathrm{C}^{\oplus n+1}\right)$.

For each $e \geq 0$ we define the Hirzebruch surface with invariant $e$ as

$$
\mathrm{F}_{e}:=\mathrm{P}\left(\mathscr{O}_{\mathrm{P}_{\mathrm{c}}^{1}} \oplus \mathcal{O}_{\mathrm{P}_{\mathrm{c}}^{1}}(-e)\right) \stackrel{\pi_{e}}{\longrightarrow} \mathrm{P}_{\mathrm{C}}^{1} \text {. }
$$

It is a ruled surface over $\mathrm{P}_{\mathrm{C}}^{1}$. In $\operatorname{Pic}\left(\mathrm{F}_{e}\right)$ we denote by $f$ and by $\xi$ the classes of a fibre of $\pi_{e}$ and of the tautological bundle $\mathscr{O}_{\mathrm{F}_{e}}(1)$.

We denote isomorphisms by $\cong$ and birational equivalences by $\approx$. For other definitions, results and notation we always refer to [6].

\section{Plane models of trigonal curves}

Recall, that the points of the locus $\mathscr{T}_{g, 0} \subseteq \mathscr{M}_{g, 0}$ represent curves carrying at least a base-point-free $g_{3}^{1}$. It is well-known that $\mathscr{T}_{g, 0}=\mathscr{M}_{g, 0}$ when $g=2,3,4$. It is classically known that $\mathscr{T}_{g, 0}$ is irreducible. Moreover, a base-point-free $g_{3}^{1}$, if any, on a curve of genus $g \geq 5$ is unique, hence complete (when $g=3$ each non-hyperelliptic curve carries infinitely many base-point-free $g_{3}^{1}$, if $g=4$ the general curve carries exactly two distinct base-point-free $g_{3}^{1}$ ).

In [12] a birational model of $\mathscr{T}_{g, 0}$ is described when $g \geq 5$. More precisely the geometric version of Riemann-Roch theorem yields that the canonical model $C_{c a n} \subseteq \mathrm{P}_{\mathrm{C}}^{g-1}$ of $C$ lies on a rational normal scroll $S$ swept out by the lines joining the points in the divisors of the unique $g_{3}^{1}$ on $C$. The surface $S$ is the image of a Hirzebruch surface $F_{e}$, via the complete linear system

$$
\left|\xi+\frac{g+e-2}{2} f\right|
$$


and $C$ is represented by an integral smooth element of

$$
\left|3 \xi+\frac{g+3 e+2}{2} f\right|
$$

that we will again denote by $C$. In particular, $g$ and $e$ have the same parity and $(g-3 e+2) / 2=\xi \cdot C_{c a n} \geq 0$, hence $0 \leq e \leq(g+2) / 3$. We have natural rational maps

$$
p_{e}:\left|3 \xi+\frac{g+3 e+2}{2} f\right| \rightarrow \mathscr{T}_{g, 0}
$$

and, due to the description above, $\mathscr{T}_{g, 0}=\bigcup_{e} \operatorname{Im}\left(p_{e}\right)$. We now go to deal with the fibres of such a map $p_{e}$.

Two elements in a fibre are curves on $\mathrm{F}_{e}$ representing the same abstract curve $C$, thus they are obtained one from the other by composing the canonical map with a suitable automorphism. Each automorphism of $C$ must fix its canonical divisor and the unique $g_{3}^{1}$ on $C$, thus it induces an automorphism of the canonical space which must fix the surface $S$. The restriction of such a projectivity to $S$ can be viewed as an automorphism of $S$, hence of the abstract surface $\mathrm{F}_{e}$ sending the linear system $\left|3 \xi+\frac{g+3 e+2}{2} f\right|$ to itself.

If $e \geq 1$, then the elements in $\operatorname{Aut}\left(\mathrm{F}_{e}\right)$ satisfy the above restriction. Indeed they must fix $\xi$, which is the unique integral divisor on $\mathrm{F}_{e}$ with negative selfintersection. Moreover, $\operatorname{Aut}\left(\mathrm{F}_{e}\right)$ fits into the following exact sequence of groups

$$
1 \longrightarrow \operatorname{Aut}\left(\mathscr{O}_{\mathrm{P}_{\mathrm{C}}^{1}} \oplus \mathcal{O}_{\mathrm{P}_{\mathrm{C}}}(-e)\right) / \mathrm{C}^{*} \longrightarrow \operatorname{Aut}\left(\mathrm{F}_{e}\right) \longrightarrow \mathrm{PGL}_{2} \longrightarrow 1
$$

(see [8], Lemmas 3, 6, 8), whence it is connected.

Let us look at the case $e=0$ so that we have to consider the automorphisms of $\mathrm{F}_{0}$ sending $\left|3 \xi+\frac{g+2}{2} f\right|$ to itself. In this case $\mathrm{F}_{0} \cong \mathrm{P}_{\mathrm{C}}^{1} \times \mathrm{P}_{\mathrm{C}}^{1}$ which is canonically isomorphic to a fixed smooth quadric $Q \subseteq \mathrm{P}_{\mathrm{C}}^{3}$ via the Segre embedding. The full group of automorphism of $F_{0}$ is not connected, since it is isomorphic to the group generated by $\mathrm{PGL}_{2} \times \mathrm{PGL}_{2}$ acting naturally onto the two rulings and by the group of order 2 generated by the involution $\mu$ exchanging the two rulings. The automorphism we are interested in are those coming from $\mathrm{PGL}_{2} \times \mathrm{PGL}_{2}$. Indeed $\mu$ sends $\left|3 \xi+\frac{g+2}{2} f\right|$ to $\left|3 f+\frac{g+2}{2} \xi\right|$ which is different from $\left|3 \xi+\frac{g+2}{2} f\right|$, due to the fact that $(g+2) / 2>3$, because $g \geq 5$ by hypothesis.

It follows that the fibres are, in any case, exactly the orbits of the natural action of the connected component $\operatorname{Aut}^{0}\left(\mathrm{~F}_{e}\right)$ of the identity inside $\operatorname{Aut}\left(\mathrm{F}_{e}\right)$ on $\left|\xi+\frac{g+3 e+2}{2} f\right|$.

We have

$$
\operatorname{dim}\left(\operatorname{Aut}^{0}\left(\mathrm{~F}_{e}\right)\right)= \begin{cases}6 & \text { if } e=0 \\ e+5 & \text { if } e \geq 1\end{cases}
$$


The assertion for $e \geq 1$ follows from Sequence 1. The assertion for $e=0$ is trivial by the description above. Moreover, each $\vartheta \in \operatorname{Aut}^{0}\left(\mathrm{~F}_{e}\right)$ in the stabilizer of a curve $C$ induces by restriction an element of $\vartheta_{\mid C} \in \operatorname{Aut}(C)$. If $\vartheta_{\mid C}$ is the identity, then $\vartheta$ would fix three points on the general fibre of $\mathrm{F}_{e}$, thus $\vartheta$ would necessarily be the identity. We conclude that the stabilizer inside $\operatorname{Aut}^{0}\left(\mathrm{~F}_{e}\right)$ of a fixed curve $C$ is isomorphic to a subgroup of $\operatorname{Aut}(C)$, hence it is finite. We conclude that

$$
\operatorname{dim}\left(\operatorname{Im}\left(p_{e}\right)\right)= \begin{cases}2 g+1 & \text { if } e=0 \\ 2 g+2-e & \text { if } e \geq 1\end{cases}
$$

The above computations prove the following well-known

LEMMA 2.1. There exists a birational equivalence

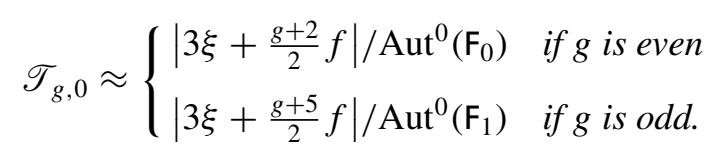

\section{Proof of the Theorem}

In this section we will prove the theorem stated in the introduction, using suitable plane models of $n$-pointed trigonal curves. We first recall the following well-known result.

Lemma 3.1. Let $m_{n}: \mathscr{M}_{g, n} \rightarrow \mathscr{M}_{g, 0}$ be the natural forgetful morphism. If $T \subseteq \mathscr{M}_{g, 0}$ is closed and irreducible, then the same is true for $m_{n}^{-1}(T) \subseteq \mathscr{M}_{g, n}$.

Proof. Let $N:=5 g-6$ and $p(t):=(g-1)(6 t-1)$. Recall that there exists a open non-empty subset of a unique irreducible component $\mathscr{H} \subseteq \mathscr{H} i l b_{p(t)}\left(\mathrm{P}_{\mathrm{C}}^{N}\right)$ such that $\mathscr{M}_{g, 0}$ is a geometric quotient of $\mathscr{H}$ modulo the natural action of $\mathrm{PGL}_{N+1}$ (see [9], Propositions 5.1, 5.3, 5.4). In particular, we have a $\mathrm{PGL}_{N+1^{-}}$ equivariant morphism $\phi: \mathscr{H} \rightarrow \mathscr{M}_{g, 0}$ whose fibre over a point representing a curve $C$ is the $\mathrm{PGL}_{N+1}$-orbit of one of its tricanonical embeddings in $\mathrm{P}_{\mathrm{C}}^{N}$. It follows that such a fibre is irreducible. Moreover, the stabilizer of such a model of $C$ coincides with $\operatorname{Aut}(C)$ which is finite, thus all the fibres have the same dimension $N^{2}+2 N$.

Let $T \subseteq \mathcal{M}_{g, 0}$ be an irreducible closed subscheme. We claim that $S:=$ $\phi^{-1}(T)$ is irreducible too. Indeed it is certainly the union $\bigcup_{i} S_{i}$ of irreducible closed components which must all be $\mathrm{PGL}_{N+1}$-invariant because the fibres are irreducible. Thus $T_{i}:=\phi\left(S_{i}\right)$ is closed too due to [9], Proposition 0.1 and Remark (6) to Proposition 0.2 . With this in mind the irreducibility of $S$ follows as in the proof of [11], Chapter I, Section 6, Theorem 8. 
At this point we can consider the incidence relation

$$
\mathscr{J}:=\left\{\left(C, p_{1}, \ldots, p_{n}\right) \in \mathscr{H} \times\left(\mathrm{P}_{\mathrm{C}}^{N}\right)^{n} \mid p_{1}, \ldots, p_{n} \in C\right\}
$$

and its projection $\psi: \mathscr{J} \rightarrow \mathscr{H}$. Such a map is projective and its fibres over a curve $C$ are isomorphic to $C^{\times n}$, thus they are irreducible and of the same dimension. It follows that the aforementioned classical result of [11], also yields the irreducibility of $R:=\psi^{-1}(S)=(\phi \circ \psi)^{-1}(T)$. Since $\mathscr{M}_{g, n}$ is a coarse moduli space for $n$-pointed curves of genus $g$, it follows the existence of a natural rational map $j: \mathscr{J} \rightarrow \mathscr{M}_{g, n}$ such that $j(R)=m_{n}^{-1}(T)$. The irreducibility of $R$ finally yields that $m_{n}^{-1}(T)$ is irreducible too.

Due to the above lemma, we deduce that the locus $\mathscr{T}_{g, n} \subseteq \mathscr{M}_{g, n}$ of $n$-pointed trigonal curves of genus $g$ is irreducible for all $n \geq 1$, since the same is true for $n=0$.

It follows that it makes sense to consider its rationality and we will distinguish two cases according to the parity of $g$. The odd genus case was essentially described in [12]. For reader's benefit we insert here the obvious modifications of the proof that one can find in the quoted paper.

To this purpose we recall the following definition (see [10], Section 2.8).

Definition 3.2. Let $G$ be an algebraic group acting on a variety $X$ and let $H \subseteq G$ be a subgroup. An irreducible subvariety $Y \subseteq X$ is called a $(G, H)$ section of $X$ if

(1) the $G$-orbits of the points of $Y$ form a dense subset of $X$;

(2) If $y \in Y, g \in H$, then $g(y) \in Y$;

(3) there is an open subvariety $Y_{0} \subseteq Y$ such that if $y \in Y_{0}$ and $g \in G$ implies $g(y) \in Y$, then $g \in H$.

The main results on $(G, H)$-sections are contained in the following lemma (see [10], Section 2.8, [4], Section 3 and the references therein).

Lemma 3.3. Let $G$ be an algebraic group acting on a variety $X, H \subseteq G$ a subgroup and let $Y \subseteq X$ be $a(G, H)$-section of $X$. Then $X / G \approx Y / H$.

\subsection{The odd genus case}

We know from the previous section that general trigonal curves with odd genus $g$ can be realised as elements of the linear system $\left|3 \xi+\frac{g+5}{2} f\right|$ onto the ruled surface $F_{1}$. Moreover, two such elements represent the same abstract curve if and only if they are in the same orbit with respect to the action of the automorphism group of $F_{1}$. Any such automorphism must necessarily fix the unique exceptional curve on $\mathrm{F}_{1}$. Let $g:=2 k+1$ so that $(g+5) / 2=k+3$. 
Contracting such an exceptional curve on $\mathrm{F}_{1}$, we obtain a plane model of $C$ as plane curve $\widehat{C}$ of degree $k+3$ with exactly one point $A$ of multiplicity $k$. With a proper choice of the coordinates $x_{0}, x_{1}, x_{2}$ in $\mathrm{P}_{\mathrm{C}}^{2}$ we can always assume that $A=E_{0}:=[1,0,0]$, so that the equation of $\widehat{C}$ is of the form

$$
v\left(x_{0}, x_{1}, x_{2}\right)=x_{0}^{3} a+x_{0}^{2} b+x_{0} c+d,
$$

where $a \in H^{0}\left(\mathrm{P}_{\mathrm{C}}^{1}, \mathscr{O}_{\mathrm{P}_{\mathrm{C}}^{1}}(k)\right) \backslash\{0\}, b \in H^{0}\left(\mathrm{P}_{\mathrm{C}}^{1}, \mathscr{O}_{\mathrm{P}_{\mathrm{C}}^{1}}(k+1)\right), c \in H^{0}\left(\mathrm{P}_{\mathrm{C}}^{1}, \mathscr{O}_{\mathrm{P}_{\mathrm{c}}^{1}}(k+\right.$ $2))$ and $d \in H^{0}\left(\mathrm{P}_{\mathrm{C}}^{1}, \mathscr{O}_{\mathrm{P}_{\mathrm{C}}^{1}}(k+3)\right)$. We denote by $V$ the subspace of $H^{0}\left(\mathrm{P}_{\mathrm{C}}^{2}\right.$, $\left.\mathcal{O}_{\mathrm{P}_{\mathrm{C}}^{2}}(k+3)\right)$ consisting of polynomials of the above form. We have

$$
\operatorname{dim}(V)=\left(\begin{array}{c}
k+5 \\
2
\end{array}\right)-\left(\begin{array}{c}
k+1 \\
2
\end{array}\right)=4 k+10=2 g+8 .
$$

The lines through $E_{0}$ cut out on $\widehat{C}$, residually to the cycle $k E_{0}$, the unique $g_{3}^{1}$ on $C$. Each automorphism in $\operatorname{Aut}\left(\mathrm{F}_{1}\right)$ fixing $C$ descends to an element in $\mathrm{PGL}_{3}$ fixing the point $E_{0}$. In particular, two elements in $V$ correspond to the same curve if and only if they are in the same orbit with respect to the action of the subgroup of $\mathrm{PGL}_{3}$ which is image, via the natural map $\mathrm{GL}_{3} \rightarrow \mathrm{PGL}_{3}$, of the subgroup $G$ of matrices of the form

$$
\gamma:=\left(\begin{array}{ccc}
\gamma_{0,0} & \gamma_{0,1} & \gamma_{0,2} \\
0 & \gamma_{1,1} & \gamma_{1,2} \\
0 & \gamma_{2,1} & \gamma_{2,2}
\end{array}\right) .
$$

Now let $p_{1}, \ldots, p_{n} \in C$ be pairwise distinct points and let $A_{1}, \ldots, A_{n}$ be their images via the projection $C \rightarrow \widehat{C}$. We have the incidence scheme

$$
\mathrm{V}_{n}:=\left\{\left(f, A_{1}, \ldots, A_{n}\right) \in V \times\left(\mathrm{P}_{\mathrm{C}}^{2}\right)^{\times n} \mid f\left(A_{i}\right)=0, i=1, \ldots, n\right\} .
$$

There exists a natural rational map $\mathrm{V}_{n} \rightarrow \mathscr{T}_{g, n}$. Due to the above discussion the fibres of such a map are exactly the $G$-orbits, thus its image has dimension

$$
\operatorname{dim}(V)+n-\operatorname{dim}(G)=2 g+1+n=\operatorname{dim}\left(\mathscr{T}_{g, n}\right) .
$$

Since $\mathscr{T}_{g, n}$ is irreducible (see Lemma 3.1), it follows that such a map is dominant, thus we obviously have the following

Proposition 3.4. $\vee_{n} / G \approx \mathscr{T}_{g, n}$ for $n \geq 0$.

We are now ready to prove the Theorem stated in the introduction when $g \geq 5$ is odd.

Proof of the Theorem when $g \geq 5$ is odd. Let $n=1$ and consider $\mathrm{W}_{1}:=\left\{\left(f, E_{1}\right) \in \mathrm{V}_{1}\right\}$, where $E_{1}:=[0,1,0]$. Then it is easy to check that 
$\mathrm{W}_{1}$ is a $(G, H)$-section of $\mathrm{V}_{1}$, where $H$ is the subgroup of $G$ consisting of matrices of the form

$$
\gamma:=\left(\begin{array}{ccc}
\gamma_{0,0} & 0 & \gamma_{0,2} \\
0 & \gamma_{1,1} & \gamma_{1,2} \\
0 & 0 & \gamma_{2,2}
\end{array}\right) .
$$

Since $\mathrm{W}_{1}$ is a linear representation of a triangular subgroup of $\mathrm{GL}_{3}$, it follows from Miyata-Vinberg Theorem (see Theorem 2.11 of [10]) that

$$
\mathscr{T}_{g, 1} \approx \mathrm{V}_{1} / G \approx \mathrm{W}_{1} / H
$$

is rational.

Let $n=2$ and consider $\mathrm{W}_{2}:=\left\{\left(f, E_{1}, E_{2}\right) \in \mathrm{V}_{2}\right\}$, where $E_{2}:=[0,0,1]$. We can now argue as above denoting by $H$ the subgroup of $G$ of matrices of the form

$$
\gamma:=\left(\begin{array}{ccc}
\gamma_{0,0} & 0 & 0 \\
0 & \gamma_{1,1} & 0 \\
0 & 0 & \gamma_{2,2}
\end{array}\right) .
$$

Finally let $3 \leq n \leq 2 g+7$. Consider $\mathrm{W}_{n}:=\left\{\left(f, E_{1}, E_{2}, E_{3}, A_{4}, \ldots, A_{n}\right) \in\right.$ $\left.\mathrm{V}_{n}\right\}$, where $E_{3}:=[1,1,1]$. There exists a natural map $\pi_{n}: \mathrm{W}_{n} \rightarrow\left(\mathrm{P}_{\mathrm{C}}^{2}\right)^{\times n-3}$ endowing $\mathrm{W}_{n}$ with a natural structure of vector bundle with typical fibre of dimension

$$
\operatorname{dim}(V)-n=4 k+10-n=2 g+8-n .
$$

As above one easily checks the birational equivalence

$$
\mathscr{T}_{g, n} \approx \mathrm{V}_{n} / G \approx \mathrm{W}_{n} / H
$$

where now $H$ is the subgroup of $G$ consisting of scalar matrices. $H$ acts on $\mathrm{W}_{n}$ leaving fixed the fibres of $\pi_{n}$ and acting on them via homotheties. Thus $\mathrm{W}_{n} / H$ turns out to be a projective bundle over $\left(\mathrm{P}_{\mathrm{C}}^{2}\right)^{\times n-3}$ with typical fibre $\mathrm{P}_{\mathrm{C}}^{2 g+7-n}$. In particular, $\mathrm{W}_{n} / H$ is rational.

\subsection{The even genus case}

We know from Section 2 that general trigonal curves with even genus $g$ can be realised as elements $C \in\left|3 \xi+\frac{g+2}{2} f\right|$ onto the ruled surface $\mathrm{F}_{0}$. Moreover, when $g \geq 6$, two such elements represent the same abstract curve if and only if they are in the same orbit with respect to the action of the $\operatorname{Aut}^{0}\left(\mathrm{~F}_{0}\right) \cong$ $\mathrm{PGL}_{2} \times \mathrm{PGL}_{2}$. Recall that $\mathrm{F}_{0} \cong Q:=\left\{x_{0} x_{3}-x_{1} x_{2}\right\} \subseteq \mathrm{P}_{\mathrm{C}}^{3}$ via the Segre embedding $\sigma: \mathrm{P}_{\mathrm{C}}^{1} \times \mathrm{P}_{\mathrm{C}}^{1} \hookrightarrow \mathrm{P}_{\mathrm{C}}^{3}$ defined by

$$
\left(\left[s_{0}, s_{1}\right],\left[t_{0}, t_{1}\right]\right) \mapsto\left(s_{0} t_{0}, s_{0} t_{1}, s_{1} t_{0}, s_{1} t_{1}\right) .
$$


Thus each automorphism in $\operatorname{Aut}^{0}\left(\mathrm{~F}_{0}\right)$ is the restriction to $Q$ of an automorphism of $\mathrm{P}_{\mathrm{C}}^{3}$ fixing $Q$.

Let $E:=[0,0,0,1]=\sigma([0,1],[0,1]) \in Q$ and consider the subset $\left|3 \xi+\frac{g+2}{2} f\right|_{E}$ consisting of divisors in $\left|3 \xi+\frac{g+2}{2} f\right|$ passing through $E$. If $\left(C, p_{1}, \ldots, p_{n}\right) \in \mathscr{T}_{g, n}$ is a general point, then we can assume that $C \subseteq Q$ is in the linear system $\left|3 \xi+\frac{g+2}{2} f\right|$. Up to an element in $\operatorname{Aut}^{0}\left(\mathrm{~F}_{0}\right) \cong \mathrm{PGL}_{2} \times \mathrm{PGL}_{2}$, we can also assume that $p_{n}=E$, hence we can always assume that $C \in$ $\left|3 \xi+\frac{g+2}{2} f\right|_{E}$.

Set $g:=2 k \geq 6$ so that $(g+2) / 2=k+1 \geq 4$ and let $\mathrm{P}_{\mathrm{C}}^{2}:=\left\{x_{3}=0\right\} \subseteq \mathrm{P}_{\mathrm{C}}^{3}$. The projection $q: Q \backslash\{E\} \rightarrow \mathrm{P}_{\mathrm{C}}^{2}$ with center $E$ is birational. More precisely it is an isomorphism from the complement in $Q$ of the two lines through $E$ and the complement in $\mathrm{P}_{\mathrm{C}}^{2}$ of the two points $E_{0}=[1,0,0]$ and $E_{1}=[0,1,0]$. It squeezes the two aforementioned lines to the two points $E_{0}$ and $E_{1}$.

Via $q$ the projective space $\left|3 \xi+\frac{g+2}{2} f\right|_{E}$ is mapped isomorphically onto the linear subsystem $\Sigma \subseteq\left|\mathscr{O}_{\mathrm{P}_{\mathrm{c}}^{2}}(k+3)\right|$ consisting of curves carrying a point of multiplicity $k \geq 3$ at $E_{0}$ and a double point at $E_{1}$. If we start with an $n$-pointed curve $\left(C, p_{1}, \ldots, p_{n-1}, E\right)$, then it is mapped via $q$ to $\left(\widehat{C}, A_{1}, \ldots, A_{n-1}\right)$, where $A_{i}:=q\left(p_{i}\right), i=1, \ldots, n-1$. The point $E$ is mapped to the intersection $A_{n}$ of the tangent line to $C$ in the point $E$ itself with the projection plane. Notice that $A_{n}$ coincides with the third intersection point of $\widehat{C}$ with the line through $E_{0}$ and $E_{1}$.

Two curves in $\Sigma$ are of the form $\widehat{C^{\prime}}$ and $\widehat{C^{\prime \prime}}$ for suitable $C^{\prime}, C^{\prime \prime} \in \mid 3 \xi+$ $\left.\frac{g+2}{2} f\right|_{E}$. Let $p_{1}^{\prime}, \ldots, p_{n-1}^{\prime}, E \in C^{\prime}$ and $p_{1}^{\prime \prime}, \ldots, p_{n-1}^{\prime \prime}, E \in C^{\prime \prime}$. Finally set $A_{i}^{\prime}:=q\left(p_{i}\right) \in \widehat{C}^{\prime}, A_{i}^{\prime \prime}:=q\left(p_{i}^{\prime \prime}\right) \in \widehat{C}^{\prime \prime}, i=1, \ldots, n-1$ and let $A_{n}^{\prime}$ and $A_{n}^{\prime \prime}$ be the third intersections of the curves $\widehat{C^{\prime}}$ and $\widehat{C^{\prime \prime}}$ with the line through $E_{0}$ and $E_{1}$, respectively.

If there exists an isomorphism $\Psi:\left(C^{\prime}, p_{1}^{\prime}, \ldots, p_{n-1}^{\prime}, E\right) \rightarrow\left(C^{\prime \prime}, p_{1}^{\prime \prime}, \ldots\right.$, $p_{n-1}^{\prime \prime}, E$ ) of their models on $Q$, then such an isomorphism must induce a projectivity of the canonical space sending the unique ruled surface containing the canonical model of $C^{\prime}$ to the unique ruled surface containing the canonical model of $C^{\prime \prime}$. Thus it induces an automorphism $\varphi \in \operatorname{Aut}\left(\mathrm{F}_{0}\right)$ restricting to $\Psi$ on $\left(C^{\prime}, p_{1}^{\prime}, \ldots, p_{n-1}^{\prime}, E\right)$. In particular, we have $\varphi(E)=E$ and $\varphi \in \operatorname{Aut}^{0}\left(\mathrm{~F}_{0}\right)$. Since the elements of Aut $\left(\mathrm{F}_{0}\right)$ are exactly the elements of the stabilizer of $Q$ inside $\mathrm{PGL}_{4}$ (see [13] and [5]) and $\varphi(E)=E$, it follows that $\varphi$ induces by projection an element $\widehat{\varphi} \in \mathrm{PGL}_{2}$ sending $\left(\widehat{C^{\prime}}, A_{1}^{\prime}, \ldots, A_{n-1}^{\prime}\right)$ to $\left(\widehat{C^{\prime \prime}}, A_{1}^{\prime \prime}, \ldots, A_{n-1}^{\prime \prime}\right)$. The projectivity $\widehat{\varphi}$ fixes $E_{0}$ and $E_{1}$, hence it must map $A_{n}^{\prime}$ to $A_{n}^{\prime \prime}$.

From now on we denote by $V$ the subspace of $H^{0}\left(\mathrm{P}_{\mathrm{C}}^{2}, \mathscr{O}_{\mathrm{P}_{\mathrm{C}}^{2}}(k+3)\right)$ corresponding to $\Sigma$. We have

$$
\operatorname{dim}(V)=\left(\begin{array}{c}
k+5 \\
2
\end{array}\right)-\left(\begin{array}{c}
k+1 \\
2
\end{array}\right)-3=4 k+7=2 g+7 .
$$


Let $G \subseteq \mathrm{GL}_{3}$ be the subgroup of matrices of the form

$$
\gamma:=\left(\begin{array}{ccc}
\gamma_{0,0} & 0 & \gamma_{0,2} \\
0 & \gamma_{1,1} & \gamma_{1,2} \\
0 & 0 & \gamma_{2,2}
\end{array}\right) .
$$

We have the incidence scheme

$\mathrm{V}_{n}:=\left\{\left(f, A_{1}, \ldots, A_{n-1}\right) \in V \times\left(\mathrm{P}_{\mathrm{C}}^{2}\right)^{\times n-1} \mid f\left(A_{i}\right)=0, i=1, \ldots, n-1\right\}$

which is trivially endowed with a natural projection rational map $\mathrm{V}_{n} \rightarrow \mathscr{T}_{g, n}$. As in the odd genus case the fibres of such a map are exactly the $G$-orbits, thus its image has dimension

$$
\operatorname{dim}(V)+n-1-\operatorname{dim}(G)=2 g+1+n=\operatorname{dim}\left(\mathscr{T}_{g, n}\right) .
$$

As in the odd genus case, we deduce that such a map is dominant, and we can state the following

Proposition 3.5. $\mathrm{V}_{n} / G \approx \mathscr{T}_{g, n}$ for $n \geq 1$.

We are now ready to prove the Theorem stated in the introduction when $g \geq 2$ is even.

Proof of the Theorem When $g \geq 6$ is even. Let $n=1$. In this case $\mathrm{V}_{1}=V$, thus it is a linear representation of the triangular group $G$. We deduce that

$$
\mathscr{T}_{g, 1} \approx \mathrm{V}_{1} / G \approx V / G
$$

is rational, again by Theorem 2.11 of [10].

If $n=2$ let us consider $\mathrm{W}_{2}:=\left\{\left(f, E_{2}\right) \in \mathrm{V}_{2}\right\}$. We can now argue as above denoting by $H$ the subgroup of $G$ of matrices of the form

$$
\gamma:=\left(\begin{array}{ccc}
\gamma_{0,0} & 0 & 0 \\
0 & \gamma_{1,1} & 0 \\
0 & 0 & \gamma_{2,2}
\end{array}\right) .
$$

Finally let $3 \leq n \leq 2 g+7$. Consider $\mathrm{W}_{n}:=\left\{\left(f, E_{2}, E_{3}, A_{3}, \ldots, A_{n-1}\right) \in\right.$ $\left.\mathrm{V}_{n}\right\}$. There exists a natural map $\pi_{n}: \mathrm{W}_{n} \rightarrow\left(\mathrm{P}_{\mathrm{C}}^{2}\right)^{\times n-3}$ endowing $\mathrm{W}_{n}$ with a natural structure of vector bundle with typical fibre of dimension

$$
\operatorname{dim}(V)-(n-1)=4 k+10-n=2 g+8-n .
$$

As above one easily checks the birational equivalence

$$
\mathscr{T}_{g, n} \approx \mathrm{V}_{n} / G \approx \mathrm{W}_{n} / H
$$


where now $H$ is the subgroup of $G$ consisting of scalar matrices. At this point one can conclude as for the odd genus case.

ACKNOWLEDGEMENTS. The author has been supported by the framework of PRIN 2008 'Geometria delle varietà algebriche e dei loro spazi di moduli', cofinanced by MIUR.

\section{REFERENCES}

1. Arbarello, E., Cornalba, M., Footnotes to a paper of Beniamino Segre, Math. Ann. 256 (1981), 341-362.

2. Casnati, G., On the rationality of moduli spaces of pointed hyperelliptic curves, Rocky Mountain J. Math. (to appear).

3. Casnati, G., Fontanari, C., On the rationality of moduli spaces of pointed curves, J. London Math. Soc. (2) 75 (2007), 582-596.

4. Dolgachev, I. V., Rationality of fields of invariants, pp. 3-16 in: Spencer J. Bloch (ed.), Algebraic Geometry, Bowdoin 1985, Proc. Symp. Pure Math. 46:2, Amer. Math. Soc., Providence, RI 1987.

5. Gizatullin, M. K., Review of the paper by Turrini, Cristina: The automorphisms of rational geometric scrolls, Istit. Lombardo Accad. Sci. Lett. Rend. A 113 (1979), 210-223, MR0622106 (83c:14029).

6. Hartshorne, R., Algebraic Geometry, Grad. Texts in Math. 52, Springer, New York 1977.

7. Ma, S., The rationality of the moduli spaces of trigonal curves of odd genus, arXiv:1012.0983.

8. Maruyama, M., On automorphism groups of ruled surfaces, J. Math. Kyoto Univ. 11 (1971), 89-112.

9. Mumford, D., Fogarty, J., Kirwan, F., Geometric Invariant Theory, 3rd edition, Ergebnisse Math. Grenzgeb. (2) 34, Springer, Berlin 1994.

10. Popov, V. L., Vinberg, E. B., Invariant theory, pp. 123-284 in: A. N. Parshin and I. R. Shafarevich (eds.), Algebraic Geometry IV, Encycl. Math. Sci. 55, Springer, Berlin 1991.

11. Shafarevich, I. R., Basic Algebraic Geometry, Grundlehren math. Wiss. 213, Springer, New York 1974.

12. Shepherd-Barron, N. I., The rationality of certain spaces associated to trigonal curves, pp. 165-171 in: Spencer J. Bloch (ed.), Algebraic Geometry, Bowdoin 1985, Proc. Symp. Pure Math. 46:1, Amer. Math. Soc., Providence, RI 1987.

13. Turrini, C., The automorphisms of rational geometric scrolls, Istit. Lombardo Accad. Sci. Lett. Rend. A 113 (1979), 210-223.

DIPARTIMENTO DI MATEMATICA

POLITECNICO DI TORINO

CORSO DUCA DEGLI ABRUZZI 24

10129 TORINO

ITALY

E-mail: gianfranco.casnati@polito.it 